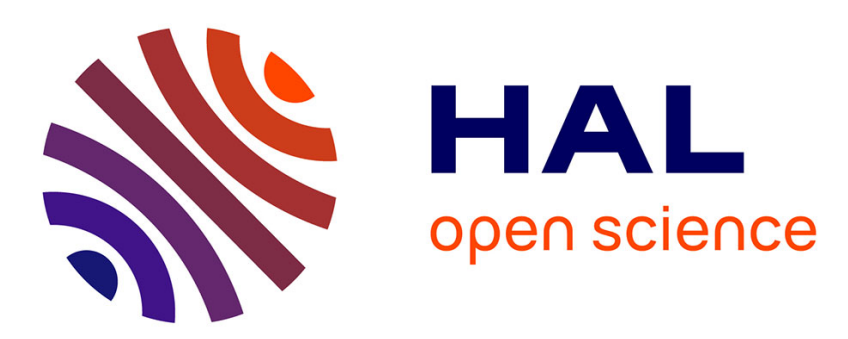

\title{
An algebraic-closure-based momentmethod for unsteady Eulerian modeling of non-isothermal particle-laden turbulent flows in very dilute regime and high Stokes number
}

Enrica Masi, Olivier Simonin

\section{To cite this version:}

Enrica Masi, Olivier Simonin. An algebraic-closure-based momentmethod for unsteady Eulerian modeling of non-isothermal particle-laden turbulent flows in very dilute regime and high Stokes number. Turbulence, Heat and Mass Transfer 7, Sep 2012, Palermo, Italy. hal-00915061

\author{
HAL Id: hal-00915061 \\ https://hal.science/hal-00915061
}

Submitted on 6 Dec 2013

HAL is a multi-disciplinary open access archive for the deposit and dissemination of scientific research documents, whether they are published or not. The documents may come from teaching and research institutions in France or abroad, or from public or private research centers.
L'archive ouverte pluridisciplinaire HAL, est destinée au dépôt et à la diffusion de documents scientifiques de niveau recherche, publiés ou non, émanant des établissements d'enseignement et de recherche français ou étrangers, des laboratoires publics ou privés. 


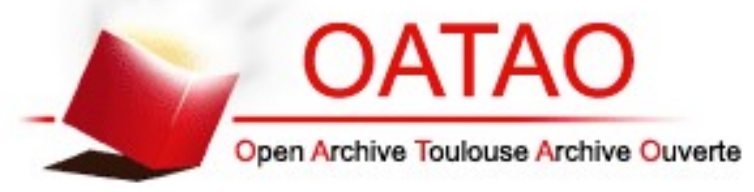

Open Archive TOULOUSE Archive Ouverte (OATAO)

OATAO is an open access repository that collects the work of Toulouse researchers and makes it freely available over the web where possible.

This is an author-deposited version published in : http://oatao.univ-toulouse.fr/ Eprints ID : 10369

To cite this version : Masi, Enrica and Simonin, Olivier An algebraicclosure-based momentmethod for unsteady Eulerian modeling of nonisothermal particle-laden turbulent flows in very dilute regime and high Stokes number. In: Turbulence, Heat and Mass Transfer 7, 24 September 2012 - 27 September 2012 (Palermo, Italy).

Any correspondance concerning this service should be sent to the repository administrator: staff-oatao@listes-diff.inp-toulouse.fr 


\title{
An algebraic-closure-based moment method for unsteady Eulerian modeling of non-isothermal particle-laden tur- bulent flows in very dilute regime and high Stokes num- ber
}

\author{
E. Masi and O. Simonin
}

Institut de Mécanique des Fluides de Toulouse, UMR CNRS/INPT/UPS 5502, Allée Camille Soula, 31400 Toulouse, France, masi@imft.fr, simonin@imft.fr

\begin{abstract}
An algebraic-closure-based moment method (ACBMM) is developed for unsteady Eulerian particle simulations coupled with direct numerical simulations (DNS) of non-isothermal fluid turbulent flows, in very dilute regime and for large Stokes numbers. It is based on a conditional statistical approach [1] which provides a local instantaneous characterization of the dynamic of the dispersed phase accounting for the effect of crossing between particle trajectories which occurs for large Stokes numbers.
\end{abstract}

\section{Introduction}

Particle-laden turbulent flows are encountered in several industrial applications and need to be modeled. In order to model the turbulence at industrial scales, Reynolds-averaged NavierStokes (RANS) methods or large-eddy simulations (LES) are usually used depending on the nature of the flow. The LES are particularly adequate for modeling unsteady turbulences of internal flows confined in complex geometries as, for instance, in combustion chambers of aero engines. For these two-phase flow applications, the entire mixture needs to be modeled and a question about the choice of the most appropriate method for modeling the particulate phase arises. In the literature, a method which has been extensively used over the years is the Eulerian-Lagrangian LES approach [2,3]. According to this approach, the turbulence is resolved by means of the LES and the particle properties are obtained by using a Lagrangian method. So far, the Eulerian-Lagrangian LES approach has presented two major limits: the prohibitive computational cost of the numerical simulation when this method is used for real industrial applications; the difficulty to model the coupling between particles and turbulent fluid flow because of the small non-resolved scales of the turbulence $[4,5]$. An alternative to the Eulerian-Lagrangian LES approach is the Eulerian-Eulerian LES approach. The latter implies that the particulate phase is described as a continuum medium at the same level as the turbulence so that a straightforward coupling between phases is possible in an Eulerian framework. This approach is considerably less expensive than a Lagrangian technique and thus suitable provided that a satisfactory level of accuracy is ensured. In the literature, several successfully models are available in the framework of the Eulerian-Eulerian RANS modeling [6, 7, 8, 9, 10]. In contrast, the Eulerian-Eulerian LES (or DNS, as direct numerical simulations) of dilute or very dilute highly inertial particle-laden turbulent flows are still a timely topic of research. Indeed, the existing DNS/LES Eulerian models $[11,12,13,14,15,16]$ have proven to be able to correctly predict the dispersed phase behaviour only for Stokes numbers smaller than the critical one ( $S t_{K}<<1$ based on the Kolmogorov timescale). In this work an appropriate model for predicting unsteady Eulerian particle simulations coupled with DNS of non-isothermal fluid turbulent flows in very dilute regime and for large Stokes numbers is proposed. It should be 
considered as the starting point for developing the Eulerian-Eulerian LES approach [17].

\section{Unsteady Eulerian Modeling}

The algebraic-closure-based moment method (ACBMM) is based on a conditional statistical approach [1] which provides a local instantaneous characterization of the dynamic of the dispersed phase accounting for the effect of crossing between particle trajectories which occurs for large Stokes numbers. This phenomenon has a great impact on the thermal characterization of the dispersed phase as well. Indeed, the occurrence of crossing trajectories entails that particles convey information of their interactions with very distant, and independent, turbulent eddies, that is with different dynamic and thermal turbulent scales. This effect involves many different velocities and temperatures in the same volume of control, violating the assumption of the uniqueness of the particle velocity and temperature distributions. An extension of the statistical approach accounting for non-isothermal conditions may be found in Ref. [18]. Particle velocity and temperature are partitioned in two contributions: i) an Eulerian particle (velocity and temperature) field, referred to as mesoscopic field, which is spatially correlated and shared by all the particles and which accounts for correlations between particles and between particles and fluid; ii) a spatially-uncorrelated particle (velocity and temperature) contribution, referred to as random uncorrelated motion (RUM) contribution, associated with each particle and resulting from the chaotic behaviour of the particles. The RUM contribution is characterized in terms of Eulerian fields of particle velocity and temperature moments; the larger is the particle inertia the more important is the RUM. The proposed ACBMM computes low-order moments of the conditional probability density function (PDF) using a set of governing equations which are derived from the PDF kinetic equation in the general frame of the kinetic theory of dilute gases. It consists of a set of three equations describing the evolution of the mesoscopic particle number density $\widetilde{n}_{p}$, the mesoscopic velocity $\widetilde{u}_{p, i}$ and the mesoscopic temperature $\widetilde{T}_{p}$, which needs to be closed by providing constitutive relations as algebraic closures. Neglecting gravity and radiant sources and assuming non-interacting and non-colliding spherical particles in translation, with a same diameter equal or smaller than the Kolmogorov lengthscale, a density much larger than that of the fluid, stationaries inter-phase heat transfers and constant heat capacity, the set of Eulerian equations is $[19,18]$

$$
\begin{aligned}
\frac{\partial \widetilde{n}_{p}}{\partial t} & +\frac{\partial \widetilde{n}_{p} \widetilde{u}_{p, i}}{\partial x_{i}}=0 \\
\frac{\partial \widetilde{n}_{p} \widetilde{u}_{p, i}}{\partial t}+\frac{\partial \widetilde{n}_{p} \widetilde{u}_{p, i} \widetilde{u}_{p, j}}{\partial x_{j}} & =-\frac{\widetilde{n}_{p}}{\widetilde{\tau}_{p}}\left(\widetilde{u}_{p, i}-u_{f, i}\right)-\frac{\partial \widetilde{n}_{p} \delta R_{p, i j}}{\partial x_{j}} \\
\frac{\partial \tilde{n}_{p} \widetilde{T}_{p}}{\partial t}+\frac{\partial \tilde{n}_{p} \tilde{u}_{p, j} \widetilde{T}_{p}}{\partial x_{j}} & =-\frac{\tilde{n}_{p}}{\widetilde{\tau}_{\theta}}\left(\widetilde{T}_{p}-T_{f}\right)-\frac{\partial \tilde{n}_{p} \delta \Theta_{p, j}}{\partial x_{j}}
\end{aligned}
$$

where $u_{f, i}$ and $T_{f}$ are the fluid velocity and temperature and $\widetilde{\tau}_{p}=\left\langle\left(1 / \tau_{p}\right) \mid \boldsymbol{x}_{p}(t)=\boldsymbol{x} ; \mathscr{H}_{f}\right\rangle^{-1}$ and $\widetilde{\tau}_{\theta}=\left\langle\left(1 / \tau_{\theta}\right) \mid \boldsymbol{x}_{p}(t)=\boldsymbol{x} ; \mathscr{H}_{f}\right\rangle^{-1}$ are the mesoscopic dynamic and thermal response times obtained by conditional ensemble averaging the particle response times

$$
\tau_{p}=\frac{4 \rho_{p} d_{p}}{3 \rho_{f} C_{D} \| \boldsymbol{u}_{p}-\mathbf{u}_{f} @ p} \| \mid \quad \tau_{\theta}=\frac{\operatorname{Pr} \rho_{p} d_{p}^{2} C_{p p}}{6 N u \mu_{f} C_{p f}} ;
$$

$\mathbf{u}_{f @ p}$ is the fluid velocity at the particle location, $\operatorname{Pr}$ is the Prandtl number, $\mu_{f}$ and $\nu_{f}$ are the dynamic and kinematic fluid viscosity respectively, $\rho_{f}$ is the density of the fluid, $\rho_{p}$ and $d_{p}$ are 
the particle density and the particle diameter, and $C_{p p} / C_{p f}$ is the particle-to-fluid heat capacity ratio. $C_{D}$ and $N u$ are the corrected drag coefficient [20] and Nusselt number [21]

$$
C_{D}=\frac{24}{R e_{p}}\left(1+0.15 R e_{p}^{0.687}\right), \quad N u=2+0.552 \operatorname{Re}_{p}^{1 / 2} \operatorname{Pr}^{1 / 3}
$$

formulated in terms of the particle Reynolds number, $R e_{p}=\| \boldsymbol{u}_{p}-\mathbf{u}_{f} @ p|| d_{p} / \nu_{f}$. The highestorder moment appearing in Eq. (2) is the second-order velocity moment referred to as RUM particle kinetic stress tensor (RUM-ST), $\delta R_{p, i j}(\boldsymbol{x}, t)=\left\langle\delta u_{p, i}(t) \delta u_{p, j}(t) \mid \boldsymbol{x}_{p}(t)=\boldsymbol{x} ; \mathscr{H}_{f}\right\rangle$. The highest-order unclosed moment in Eq. (3) is the velocity-temperature correlation $\delta \Theta_{p, i}(\boldsymbol{x}, t)=$ $\left\langle\delta u_{p, i}(t) \delta T_{p}(t) \mid \boldsymbol{x}_{p}(t)=\boldsymbol{x} ; \mathscr{H}_{f}\right\rangle$ referred to as RUM particle heat flux (RUM-HF) for the sake of simplicity (although this term has no longer units of a heat flux since the particle mass and heat capacity were removed because constant quantities). The first term on the right hand side (r.h.s.) of Eq. (2) accounts for the effects of the drag force and the second term is the transport due to the RUM-ST. The first term on the r.h.s. of Eq. (3) represents the interphase heat exchanges and the second contribution is the transport of the mesoscopic temperature due to the RUM-HF. At the second order, the modeling leads to an equation for the RUM-ST [22]

$$
\frac{\partial \widetilde{n}_{p} \delta R_{p, i j}}{\partial t}+\frac{\partial \widetilde{n}_{p} \delta R_{p, i j} \tilde{u}_{p, k}}{\partial x_{k}}=-2 \frac{\widetilde{n}_{p}}{\widetilde{\tau}_{p}} \delta R_{p, i j}-\widetilde{n}_{p} \delta R_{p, j k} \frac{\partial \widetilde{u}_{p, i}}{\partial x_{k}}-\widetilde{n}_{p} \delta R_{p, i k} \frac{\partial \widetilde{u}_{p, j}}{\partial x_{k}}-\frac{\partial}{\partial x_{k}} \widetilde{n}_{p} \delta Q_{p, i j k},
$$

and an equation for the RUM-HF [23]

$$
\begin{aligned}
\frac{\partial}{\partial t} \tilde{n}_{p} \delta \Theta_{p, i}+\frac{\partial}{\partial x_{j}} \tilde{n}_{p} \tilde{u}_{p, j} \delta \Theta_{p, i}= & -\tilde{n}_{p}\left(\frac{1}{\widetilde{\tau}_{p}}+\frac{1}{\widetilde{\tau}_{\theta}}\right) \delta \Theta_{p, i}-\tilde{n}_{p} \delta \Theta_{p, j} \frac{\partial \tilde{u}_{p, i}}{\partial x_{j}}-\tilde{n}_{p} \delta R_{p, i j} \frac{\partial \widetilde{T}_{p}}{\partial x_{j}} \\
& -\frac{\partial}{\partial x_{j}} \tilde{n}_{p} \delta \Delta_{p, i j} .
\end{aligned}
$$

The highest-order moments appearing in Eqs. (6)-(7) are third-order RUM correlations defined by the conditional ensemble average as $\delta Q_{p, i j k}(\boldsymbol{x}, t)=\left\langle\delta u_{p, i}(t) \delta u_{p, j}(t) \delta u_{p, k}(t) \mid \boldsymbol{x}_{p}(t)=\boldsymbol{x} ; \mathscr{H}_{f}\right\rangle$ and $\delta \Delta_{p, i j}(\boldsymbol{x}, t)=\left\langle\delta u_{p, i}(t) \delta u_{p, j}(t) \delta T_{p}(t) \mid \boldsymbol{x}_{p}(t)=\boldsymbol{x} ; \mathscr{H}_{f}\right\rangle$. The first term on the r.h.s. of Eq. (6) represents the dissipation of the RUM-ST while second and third terms are productions by mesoscopic velocity gradients. The first term on the r.h.s. of Eq. (7) accounts for the dissipation of the RUM-HF due to heat and momentum transfers and second and third terms are productions by both mesoscopic velocity and temperature gradients. In the framework of the ACBMM approach proposed in this study, Eqs. (6) and (7) are not resolved but rather used for the development of the second-order moment closures. Preliminary two-dimensional Eulerian-Eulerian DNS using modeled RUM-ST in conjunction with resolved RUM-HF may be found in Ref. [24]. With the purpose of deriving algebraic closures, the equation of the evolution of the RUM kinetic energy $\left(\delta \theta_{p}(\boldsymbol{x}, t)=0.5\left\langle\delta u_{p, i}(t) \delta u_{p, i}(t) \mid \boldsymbol{x}_{p}(t)=\boldsymbol{x} ; \mathscr{H}_{f}\right\rangle\right)$ and the RUM temperature variance $\left(\delta \theta_{\theta}(\boldsymbol{x}, t)=0.5\left\langle\delta T_{p}(t) \delta T_{p}(t) \mid \boldsymbol{x}_{p}(t)=\boldsymbol{x} ; \mathscr{H}_{f}\right\rangle\right)$ are also written [19, 23]:

$$
\begin{gathered}
\frac{\partial \widetilde{n}_{p} \delta \theta_{p}}{\partial t}+\frac{\partial \widetilde{n}_{p} \delta \theta_{p} \tilde{u}_{p, m}}{\partial x_{m}}=-\widetilde{n}_{p} \delta R_{p, n m} \frac{\partial \widetilde{u}_{p, n}}{\partial x_{m}}-2 \frac{\widetilde{n}_{p}}{\tilde{\tau}_{p}} \delta \theta_{p}-\frac{1}{2} \frac{\partial}{\partial x_{m}} \widetilde{n}_{p} \delta Q_{p, n n m}, \\
\frac{\partial \widetilde{n}_{p} \delta \theta_{\theta}}{\partial t}+\frac{\partial \widetilde{n}_{p} \delta \theta_{\theta} \tilde{u}_{p, m}}{\partial x_{m}}=-\widetilde{n}_{p} \delta \Theta_{p, m} \frac{\partial \widetilde{T}_{p}}{\partial x_{m}}-2 \frac{\widetilde{n}_{p}}{\tilde{\tau}_{\theta}} \delta \theta_{\theta}-\frac{1}{2} \frac{\partial}{\partial x_{m}} \widetilde{n}_{p} \delta \Omega_{p, m} .
\end{gathered}
$$

The last term in Eq. (9) is defined as $\delta \Omega_{p, m}(\boldsymbol{x}, t)=\left\langle\delta u_{p, m}(t) \delta T_{p}(t) \delta T_{p}(t) \mid \boldsymbol{x}_{p}(t)=\boldsymbol{x} ; \mathscr{H}_{f}\right\rangle$. The ACBMM bases its efficiency and accuracy on the algebraic closures provided for modeling the RUM-ST and the RUM-HF; in this study, this concern is addressed. 


\section{Modeling The RUM Particle Kinetic Stress Tensor (RUM-ST)}

The RUM stress tensor is composed of spherical and deviatoric contributions

$$
\delta R_{p, i j}=\frac{1}{3} \delta R_{p, k k} \delta_{i j}+\delta R_{p, i j}^{*}=\frac{2}{3} \delta \theta_{p} \delta_{i j}+\delta R_{p, i j}^{*} .
$$

The spherical part may be obtained by means of an additional transport equation $[19,25]$ while the deviatoric contribution needs to an algebraic closure. In this work the deviatoric RUM-ST is modeled by using a polynomial representation for tensor functions [23]. The polynomial representation is used in the framework of an assumption of equilibrium of the local anisotropy. Indeed, one of the most important finding of this study was to observe that the RUM-ST is a self-similar tensor which means that its temporal and spatial evolutions are related to that of its trace, involving equilibrium of anisotropy. Such an equilibrium leads to an algebraic stress model (ASM) which is an implicit and nonlinear system of equations referred to as $2 \Phi A S M$ (by analogy with turbulence models, see, e.g. [26] and references cited in). It is obtained defining a local RUM anisotropy tensor as

$$
b_{p, i j}^{*}=\frac{\delta R_{p, i j}}{2 \delta \theta_{p}}-\frac{1}{3} \delta_{i j}
$$

Then the equilibrium involves

$$
\frac{D}{D t} b_{p, i j}^{*}=0 \quad \text { with } \quad \frac{D}{D t}=\frac{\partial}{\partial t}+\widetilde{u}_{p, j} \frac{\partial}{\partial x_{j}},
$$

which using the definition (11) gives the relation

$$
\frac{D}{D t} \delta R_{p, i j}=\frac{\delta R_{p, i j}}{\delta \theta_{p}} \frac{D}{D t} \delta \theta_{p}
$$

Injecting Eqs. (6) and (8) into Eq. (13), and assuming equality between left hand side (1.h.s.) and r.h.s. third-order correlations, the equation takes the form

$$
\delta R_{p, i j}\left(-\frac{\delta R_{p, n m}}{2 \delta \theta_{p}} \frac{\partial \widetilde{u}_{p, n}}{\partial x_{m}}\right)=-\frac{1}{2} \delta R_{p, j k} \frac{\partial \widetilde{u}_{p, i}}{\partial x_{k}}-\frac{1}{2} \delta R_{p, i k} \frac{\partial \widetilde{u}_{p, j}}{\partial x_{k}} .
$$

Defining the mesoscopic particle rate-of-strain and vorticity tensors as, respectively,

$$
S_{p, i j}=\frac{1}{2}\left(\frac{\partial \widetilde{u}_{p, i}}{\partial x_{j}}+\frac{\partial \widetilde{u}_{p, j}}{\partial x_{i}}\right), \quad \Omega_{p, i j}=\frac{1}{2}\left(\frac{\partial \widetilde{u}_{p, i}}{\partial x_{j}}-\frac{\partial \widetilde{u}_{p, j}}{\partial x_{i}}\right)
$$

and using the decomposition of $S_{p, i j}$ in deviatoric and spherical contributions $S_{p, i j}=S_{p, i j}^{*}+$ $\frac{1}{3} S_{p, k k} \delta_{i j}$ and the RUM anisotropy definition, Eq. (14) may be rearranged as

$$
\begin{aligned}
b_{p, i j}^{*}\left(-2 b_{p, n m}^{*} S_{p, n m}^{*}\right)= & -\frac{2}{3} S_{p, i j}^{*}-\left(b_{p, i k}^{*} S_{p, k j}^{*}+S_{p, i k}^{*} b_{p, k j}^{*}-\frac{2}{3} b_{p, n m}^{*} S_{p, n m}^{*} \delta_{i j}\right) \\
& +\left(b_{p, i k}^{*} \Omega_{p, k j}-\Omega_{p, i k} b_{p, k j}^{*}\right)
\end{aligned}
$$

which represents the implicit nonlinear $2 \Phi A S M$ model proposed for closing the RUM-ST. Explicit solutions are then provided using techniques well known in turbulence [27, 28, 29]. Hereinafter, bold notation denotes three-dimensional second-rank tensors, curly brackets $\{$.$\} repre-$ sent the tensor trace and the asterisk means traceless tensor. The matrix multiplication is then 
defined in a matrix notation as $\mathbf{C}=\mathbf{A B}=A_{i k} B_{k j}=C_{i j}$ and $\mathbf{B}^{2}=\mathbf{B B}$. According to Pope [30], the anisotropy tensor may be expressed using a polynomial representation for tensor functions $\mathbf{b}^{*}=\sum_{\varsigma} G_{\varsigma} \mathbf{T}^{(\varsigma)}$ which represents the linear combination of a set of non-dimensional independent, symmetric and deviatoric second-order tensors $\mathrm{T}^{(\varsigma)}$, using scalar coefficients $G_{\varsigma}$ which are functions of the invariants of the dimensionless $\mathbf{S}^{+}$and $\Omega^{+}$. Using the CayleyHamilton theorem, Pope [30] showed that a set of ten $(\varsigma=10)$ tensors $\mathbf{T}^{(\varsigma)}$ is needed to form an integrity basis [31] in order to express every symmetric deviatoric three-dimensional secondorder tensor formed by $\mathrm{S}^{+}$and $\Omega^{+}$; the problem then reduces to model the ten coefficients $G_{\varsigma}$ (see, e.g., Refs. [27, 29]). However, the integrity basis and coefficients associated with may be reduced if some approximations are introduced. Using a two-dimensional flow approximation (three tensor basis) Girimaji [28] developed a self-consistent solution technique in order to model the Reynolds stresses in turbulent flows. In this work, we will use the same technique applied to our $2 \Phi A S M$ model. The algebraic, implicit and nonlinear system for the dispersed phase is rewritten using the Girimaji's notation as

$$
\mathbf{b}^{*}\left(L_{1}^{0}-L_{1}^{1}\left\{\mathbf{b}^{*} \mathbf{S}^{+}\right\}\right)=L_{2} \mathbf{S}^{+}+L_{3}\left(\mathbf{b}^{*} \mathbf{S}^{+}+\mathbf{S}^{+} \mathbf{b}^{*}-\frac{2}{3}\left\{\mathbf{b}^{*} \mathbf{S}^{+}\right\} \mathbf{I}\right)-L_{4}\left(\mathbf{b}^{*} \boldsymbol{\Omega}^{+}-\Omega^{+} \mathbf{b}^{*}\right)
$$

where $L_{1}^{0}=0, L_{1}^{1}=2, L_{2}=-\frac{2}{3}, L_{3}=-1$ and $L_{4}=-1, \mathbf{S}^{+}$and $\Omega^{+}$are dimensionless tensors by the quantity $I I_{S}^{1 / 2}=\left\{\mathbf{S}^{* 2}\right\}^{\mathbf{1} / \mathbf{2}}$ and $\mathbf{I}$ is the identity matrix. According to Girimaji [28], the general representation of the anisotropy tensor under the two-dimensional approximation is

$$
\mathbf{b}^{*}=\sum_{\varsigma=1}^{3} G_{\varsigma} \mathbf{T}^{(\varsigma)}=G_{1} \mathbf{S}^{+}+G_{2}\left(\mathbf{S}^{+} \mathbf{\Omega}^{+}-\mathbf{\Omega}^{+} \mathbf{S}^{+}\right)+G_{3}\left(\mathbf{S}^{+2}-\frac{1}{3}\left\{\mathbf{S}^{+2}\right\} \mathbf{I}\right),
$$

where the three coefficients are functions of the two invariants $\eta_{1}=\left\{\mathbf{S}^{+2}\right\}$ and $\eta_{2}=\left\{\boldsymbol{\Omega}^{+2}\right\}$. Using Eq. (18) and the two-dimensional hypothesis, the contracted product $b_{p, i j}^{*} S_{p, i j}^{+}$may be written as

$$
b_{p, i j}^{*} S_{p, i j}^{+}=G_{1} S_{p, i j}^{+} S_{p, i j}^{+}=G_{1} \eta_{1} .
$$

Inserting equations (18) and (19) into the system (17) leads to

$$
\begin{aligned}
& {\left[G_{1} \mathbf{S}^{+}+G_{2}\left(\mathbf{S}^{+} \boldsymbol{\Omega}^{+}-\boldsymbol{\Omega}^{+} \mathbf{S}^{+}\right)+G_{3}\left(\mathbf{S}^{+2}-\frac{1}{3}\left\{\mathbf{S}^{+2}\right\} \mathbf{I}\right)\right]\left(L_{1}^{0}-\eta_{1} L_{1}^{1} G_{1}\right)=} \\
& \left(L_{2}+\frac{1}{3} \eta_{1} L_{3} G_{3}-2 \eta_{2} L_{4} G_{2}\right) \mathbf{S}^{+}+2 L_{3} G_{1}\left(\mathbf{S}^{+2}-\frac{1}{3}\left\{\mathbf{S}^{+2}\right\} \mathbf{I}\right)-L_{4} G_{1}\left(\mathbf{S}^{+} \boldsymbol{\Omega}^{+}-\boldsymbol{\Omega}^{+} \mathbf{S}^{+}\right) .
\end{aligned}
$$

Then, comparison between homogeneous 1.h.s. and r.h.s. terms in Eq. (20) leads to explicit solutions for the unknown coefficients. In our particular case in which $L_{1}^{0}=0$, the coefficients $G_{2}$ and $G_{3}$ are directly obtained as follows

$$
G_{2}=\frac{L_{4}}{\eta_{1} L_{1}^{1}}, \quad G_{3}=-\frac{2 L_{3}}{\eta_{1} L_{1}^{1}}
$$

and $G_{1}$ reduces to the solution of a pure quadratic

$$
G_{1}^{2}=-\frac{1}{\eta_{1} L_{1}^{1}}\left[L_{2}+\frac{1}{3} \eta_{1} L_{3} G_{3}-2 \eta_{2} L_{4} G_{2}\right]
$$

Eq. (22) with the "L" coefficients leads to $G_{1}= \pm \sqrt{2 \eta_{1}+2 \eta_{2}} / 2 \eta_{1}$, which admits real solutions only for $\eta_{1}+\eta_{2} \geq 0$. Unfortunately, as $\eta_{1}$ and $\eta_{2}$ have positive and negative sign respectively, 

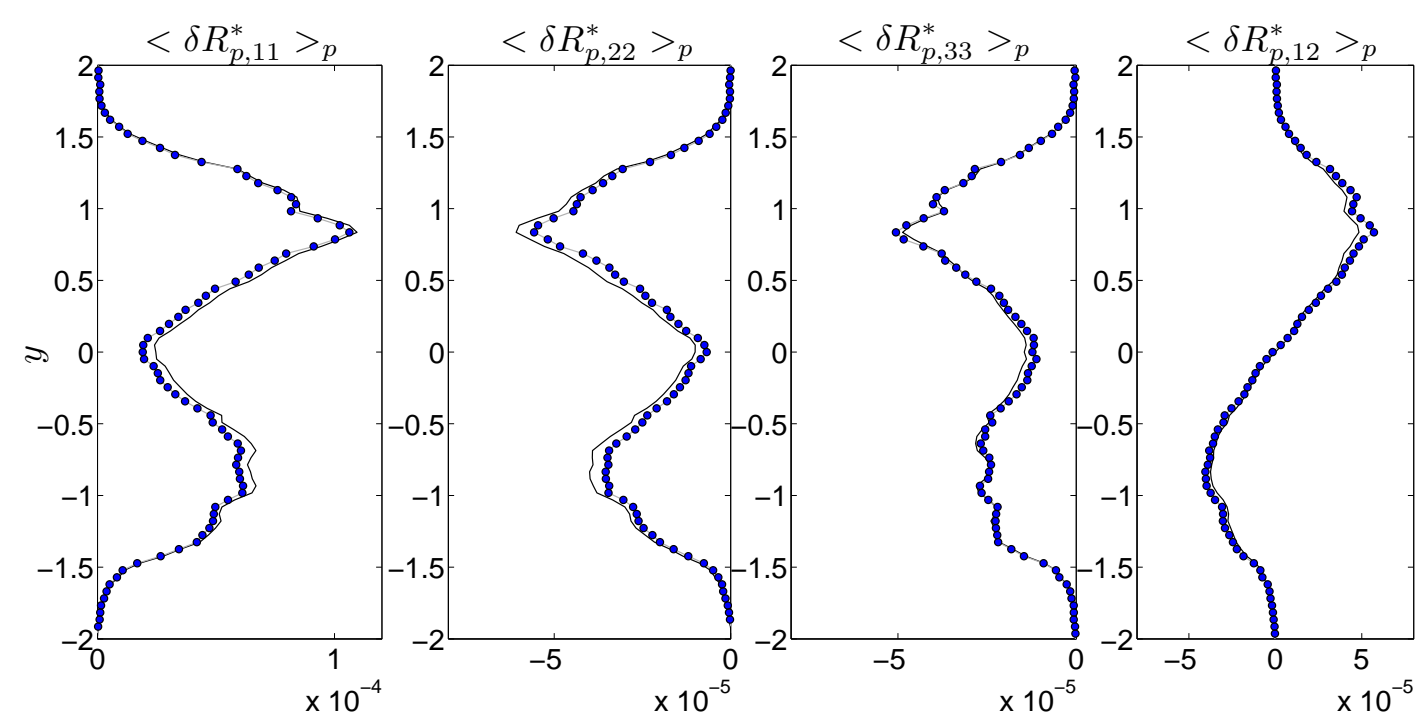

Figure 1: Profiles of actual (solid line) and modeled (line with symbols) mean deviatoric RUM stresses, at the time $t=6.2 T_{f @ p}$.

real solutions are not ensured. In order to use this model, local negative values of the discriminant are set to zero. The legitimacy of such an approximation was investigated in Ref. [23]; it was observed that negative values correspond to small magnitudes of both the particle rateof-strain and vorticity tensors justifying the approximation $G_{1}=0$. Concerning the sign of the coefficient $G_{1}$, according to Eq. (19) and the definition of the normalized production of the RUM particle kinetic energy by shear $\left(-\left\{\mathbf{b}^{*} \mathbf{S}^{*}\right\}\right.$, from Eq. (8)), in the simplest case it should be taken as negative. Negative sign for $G_{1}$ is obtained in turbulence, in the domain of applicability of an equilibrium assumption of anisotropy [28]. However, for the dispersed phase interacting with turbulent flows, it is usual to have reverse energy exchanges from the RUM to the mesoscopic contribution $[17,18]$ corresponding to a reverse sign of the first-order coefficient $G_{1}$. In the special case in which the deviatoric RUM-ST and the deviatoric particle rate-of-strain are axisymmetric tensors (as reported in Refs. [23, 32]), assuming that the principal direction of the RUM-ST is aligned with the most extensive or compressive direction of the particle rateof-strain, then the sign of the contracted product $b_{p, i j}^{*} S_{p, i j}^{+}$, which is the same of $G_{1}$, is given by the sign of the third invariant of the particle rate-of-strain tensor defined as $\eta_{3}=\left\{\mathbf{S}^{+\mathbf{3}}\right\}$. In this case the solution becomes $G_{1}=\operatorname{sign}\left(\eta_{3}\right) \sqrt{2 \eta_{1}+2 \eta_{2}} / 2 \eta_{1}$. Injecting the coefficients $G_{1}$, $G_{2}, G_{3}$ into Eq. (18) leads to an explicit solution for the RUM anisotropy tensor and thus for the RUM-ST. Hereinafter, this model will be referred to as $2 \Phi E A S M$. Results from preliminary isothermal Eulerian-Eulerian DNS using 2థEASM may be found in Ref. [33].

\section{Modeling The RUM Particle Heat Flux (RUM-HF)}

In a similar way, the RUM-HF is modeled assuming similarity between the evolution of the RUM heat flux and that of the square root correlation between the RUM kinetic energy $\left(\delta \theta_{p}\right)$ and the RUM temperature variance $\left(\delta \theta_{\theta}\right)$, which involves the equilibrium of the normalized heat flux. This assumption may be written as

$$
\frac{D}{D t} \frac{\delta \Theta_{p, i}}{\sqrt{\delta \theta_{p} \delta \theta_{\theta}}}=0
$$



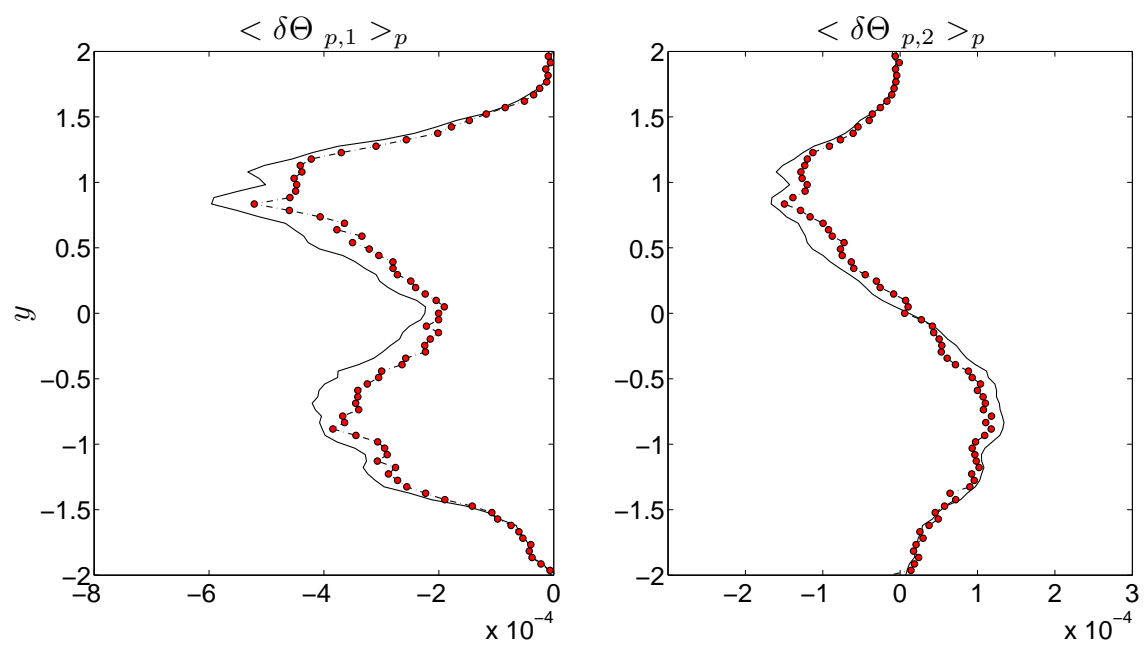

Figure 2: Profiles of actual (solid line) and modeled (line with symbols) mean streamwise and normalwise RUM-HF components, at the time $t=6.2 T_{f @ p}$.

which developed leads to

$$
\frac{1}{\sqrt{\delta \theta_{p} \delta \theta_{\theta}}} \frac{D}{D t} \delta \Theta_{p, i}=\frac{1}{2} \frac{\delta \Theta_{p, i}}{\sqrt{\delta \theta_{p} \delta \theta_{\theta}}} \frac{1}{\delta \theta_{p}} \frac{D}{D t} \delta \theta_{p}+\frac{1}{2} \frac{\delta \Theta_{p, i}}{\sqrt{\delta \theta_{p} \delta \theta_{\theta}}} \frac{1}{\delta \theta_{\theta}} \frac{D}{D t} \delta \theta_{\theta} .
$$

Then injecting Eqs. (7), (8) and (9) into Eq. (24) and assuming equality between 1.h.s. and r.h.s. diffusion terms, the equation takes the form

$$
\frac{\delta \Theta_{p, i}}{\sqrt{\delta \theta_{p} \delta \theta_{\theta}}}\left(-\frac{1}{2} \frac{\delta \Theta_{p, l}}{\delta \theta_{\theta}} \frac{\partial \widetilde{T}_{p}}{\partial x_{l}}-\frac{1}{2} \frac{\delta R_{p, k l}}{\delta \theta_{p}} \frac{\partial \widetilde{u}_{p, k}}{\partial x_{l}}\right)=-\frac{\delta R_{p, i l}}{\sqrt{\delta \theta_{p} \delta \theta_{\theta}}} \frac{\partial \widetilde{T}_{p}}{\partial x_{l}}-\frac{\delta \Theta_{p, l}}{\sqrt{\delta \theta_{p} \delta \theta_{\theta}}} \frac{\partial \widetilde{u}_{p, i}}{\partial x_{l}},
$$

which may be rearranged as follows

$$
\delta H_{p, i}\left(-\frac{1}{2} \delta H_{p, l} \tilde{K}_{p, l}-b_{p, k l}^{*} S_{p, k l}^{*}\right)=-2\left(b_{p, i l}^{*}+\frac{1}{3} \delta_{i l}\right) \tilde{K}_{p, l}-\left(S_{p, i l}^{*}+\Omega_{p, i l}\right) \delta H_{p, l},
$$

by defining the quantities

$$
\delta H_{p, i}=\frac{\delta \Theta_{p, i}}{\sqrt{\delta \theta_{p} \delta \theta_{\theta}}}, \quad \tilde{K}_{p, i}=\sqrt{\frac{\delta \theta_{p}}{\delta \theta_{\theta}}} \frac{\partial \widetilde{T}_{p}}{\partial x_{i}} .
$$

A fully dimensionless system may be obtained using non-dimensionalized quantities $\tilde{K}_{p, i}, S_{p, i j}^{*}$ and $\Omega_{p, i j}$ by the same inverse timescale, then referred to as $\widetilde{K}_{p, i}^{+}, S_{p, i j}^{+}$and $\Omega_{p, i j}^{+}$. Eq. (26) is an implicit and nonlinear particle algebraic heat flux model (AHFM), referred to as $2 \Phi A H F M$ (by analogy with turbulence models). Explicit self-consistent solutions, 2बEAHFM, are then provided using a technique suggested for turbulent heat flux by Wikström et al. [34] and by introducing a regularization procedure for ensuring non-singular solutions during the process of matrix inversion. Eq. (26) was indeed rearranged in a similar way that the analogous equation of Wikström et al. [34] in order to straightforwardly apply their technique to our 2ФAHFM. Using the same notation as in Ref. [34], we define the scalar quantity into the parentheses on the 1.h.s. of Eq. (26) as

$$
N_{\theta}=-\frac{1}{2} \delta H_{p, l} \tilde{K}_{p, l}^{+}-b_{p, k l}^{*} S_{p, k l}^{+} .
$$


The system (26) may then be solved by inverting the matrix

$$
A_{p, i j}=N_{\theta} \delta_{i j}+S_{p, i j}^{+}+\Omega_{p, i j}^{+}
$$

obtaining the explicit solutions

$$
\delta H_{p, i}=-2 A_{p, i j}^{-1}\left(b_{p, j l}^{*}+\frac{1}{3} \delta_{j l}\right) \tilde{K}_{p, l}^{+} .
$$

According to the Cayley-Hamilton theorem, an analytic expression for $\mathbf{A}^{-1}$ may be written (see, e.g., Ref. [35] ) and the operation of matrix inversion is ensured provided that $\mathbf{A}$ admits real non-zero eigenvalues so to avoid the occurrence of singularities when its determinant (denominator of the analytical solution) vanishes. The technique of Wikström et al. [34] involves injecting the relation of the flux (Eq. 30 in our case) into the definition of $N_{\theta}$ (Eq. 28 in our case), replacing $\mathbf{A}^{-1}$ by its analytical expression. For fully three-dimensional flows, this leads to a fourth-order polynomial in $N_{\theta}$ difficult to handle. An alternative is to resolve a thirdorder polynomial corresponding to a two-dimensional flow approximation and then using the solution for three-dimensional flows as well [34]. Assuming two-dimensional flows (but using three-dimensional tensors) the analytic expression for the inverse of the matrix is

$$
\mathbf{A}^{-1}=\frac{N_{\theta} \mathbf{I}-\left(\mathbf{S}^{+}+\mathbf{\Omega}^{+}\right)}{N_{\theta}^{2}-\frac{1}{2}\left(\eta_{1}+\eta_{2}\right)}
$$

Injecting Eq. (31) into Eq. (30) and the latter into the definition (28) the following third-order polynomial is obtained

$$
2 N_{\theta}^{3}+2\left\{\mathbf{b}^{*} \mathbf{S}^{+}\right\} N_{\theta}^{2}-\left(Q_{1}+R_{1}\right) N_{\theta}-\left\{\mathbf{b}^{*} \mathbf{S}^{+}\right\} Q_{1}+R_{2}=0,
$$

where $Q_{1}=\eta_{1}+\eta_{2}$ and the terms $R_{1}$ and $R_{2}$ are, respectively,

$$
\begin{aligned}
& R_{1}=2\left\{\left(b_{p, i l}^{*}+\frac{1}{3} \delta_{i l}\right) \tilde{K}_{p, l}^{+} \tilde{K}_{p, i}^{+}\right\} \\
& R_{2}=2\left\{\left(S_{p, i j}^{+}+\Omega_{p, i j}^{+}\right)\left(b_{p, j l}^{*}+\frac{1}{3} \delta_{j l}\right) \tilde{K}_{p, l}^{+} \tilde{K}_{p, i}^{+}\right\} .
\end{aligned}
$$

A unique real solution is then ensured using transcendental functions as in Ref. [34]. At this point, we need to address the concern of stable solutions by ensuring the determinant of the matrix does not vanish and singularities do not occur. From the a priori analysis, we observed that the determinant is mainly positive and that negative values correspond to small magnitudes of it. In order to avoid singular solutions the determinant should not change its sign and the following condition should be satisfied

$$
\operatorname{det}(\mathbf{A})=N_{\theta}^{2}-\frac{1}{2}\left(\eta_{1}+\eta_{2}\right)>0
$$

while, in general, it is not. Solutions proposed in Ref. [34] for turbulent flows do not apply to the dispersed phase and a regularization procedure is therefore necessary. The reciprocal of the determinant is re-written as follows

$$
\operatorname{det}(\mathbf{A})^{-1}=\frac{1}{N_{\theta}^{2}-\frac{1}{2}\left(\eta_{1}+\eta_{2}\right)}=\frac{2 /\left(2 N_{\theta}^{2}\right)}{1-\eta^{2} /\left(2 N_{\theta}^{2}\right)+\xi^{2} /\left(2 N_{\theta}^{2}\right)}=\frac{2 /\left(2 N_{\theta}^{2}\right)}{1+\xi^{2} /\left(2 N_{\theta}^{2}\right)-x^{2}}
$$



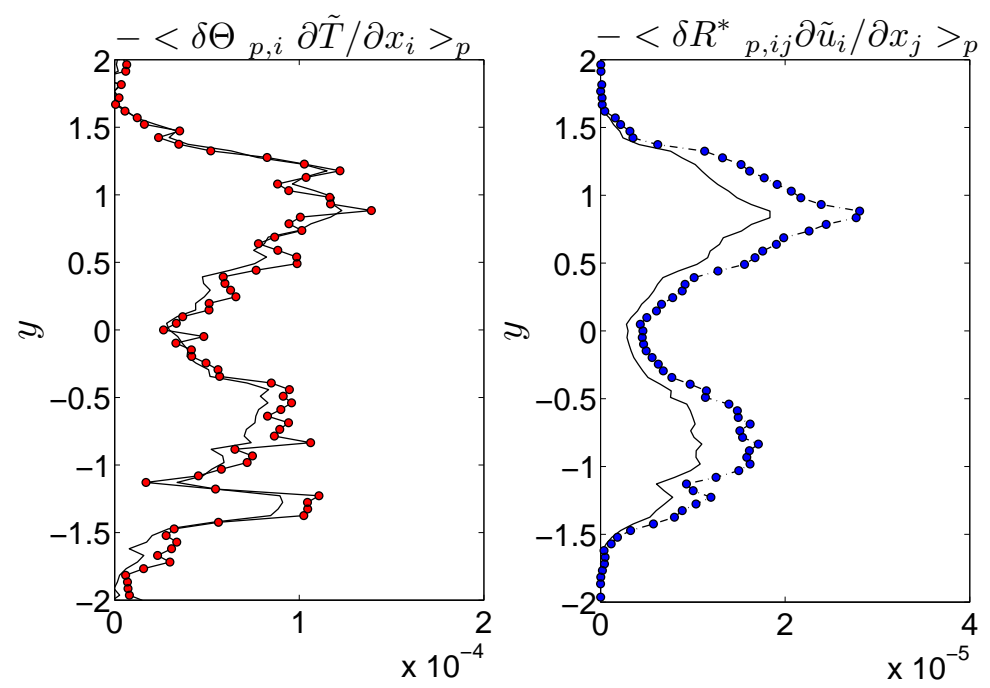

Figure 3: Mean profiles of actual (solid line) and modeled (line with symbols) RUM temperature variance production (left) and RUM kinetic energy production (right), at the time $t=6.2 T_{f} @$.

where $\eta=\sqrt{\eta_{1}}, \xi=\sqrt{-\eta_{2}}$ and $x^{2}=\eta^{2} /\left(2 N_{\theta}^{2}\right)$. Then, using the first order MacLaurin series of the function $1 /\left(1+x^{2}\right)$ we write $x^{2} \approx 1-1 /\left(1+x^{2}\right)$ which inserted into Eq. (35) leads to

$$
\operatorname{det}(\mathbf{A})^{-1}=\frac{2\left(2 N_{\theta}^{2}+\eta^{2}\right)}{\left(2 N_{\theta}^{2}\right)^{2}+\xi^{2}\left(2 N_{\theta}^{2}+\eta^{2}\right)} .
$$

The stability is thus ensured as the denominator of Eq. (36) never vanishes for $N_{\theta}$ and $\xi^{2}$ or $\eta^{2} \neq 0$. The same regularization procedure was used by Gatski and Speziale [27] for modeling the Reynolds stress tensor in the framework of the EASM approach. Hereinafter, explicit regularized solutions for the RUM-HF will be referred to as $2 \Phi E A H F M$.

\section{A Priori Analysis}

An evaluation of the ACBMM by assessing the proposed algebraic closures is given by an a priori analysis using particle Eulerian fields which are extracted from a Eulerian-Lagrangian DNS of a temporal particle-laden non-isothermal turbulent planar jet. The numerical simulation corresponds to the dispersion of a cold particle-laden jet into a hot homogeneous isotropic decaying turbulence [36]. The simulation domain is a cube of length size $L_{b o x}=2 \pi L_{r e f}$ and mesh $128^{3}$ cells. The initial gas velocity has hyperbolic-tangent profile supplemented with statistically homogeneous and isotropic velocity fluctuations [37]. The initial gas temperature has hyperbolic-tangent mean profile and zero fluctuations. As we assume very dilute regime, collisions and turbulence modulation are not accounted for. Within the slab, of width $d=$ $0.25 L_{\text {box }}, 13$ millions of solid particles are randomly embedded at the same mean velocity and temperature as the carrier flow and zero fluctuations. The simulation corresponds to a Stokes number $S t \sim 1$ computed over a characteristic timescale of the turbulence seen by the particles representing an estimate of the integral Lagrangian timescale $T^{L}$ in mean-sheared flows [38]. The Reynolds number based on the energetic lengthscale is $R e_{L e} \sim 73$. The initial set of dimensionless parameters is: kinematics viscosity $\nu_{f}=1.82 \mathrm{e}-4 u_{r e f} L_{r e f}$, turbulent kinetic energy $q_{f}^{2}=3.37 \mathrm{e}-4 u_{r e f}^{2}$, dissipation $\epsilon_{f}=3.78 \mathrm{e}-5 u_{r e f}^{3} / L_{r e f}$, jet mean velocity $U_{f}=$ $0.15 u_{r e f}$, jet rms velocity $u_{f}^{\prime}=0.015 u_{r e f}$, particle diameter $d_{p} / \Delta_{x}=0.01$. The particle Eulerian database is obtained from the Eulerian-Lagrangian DNS using the projection procedure 

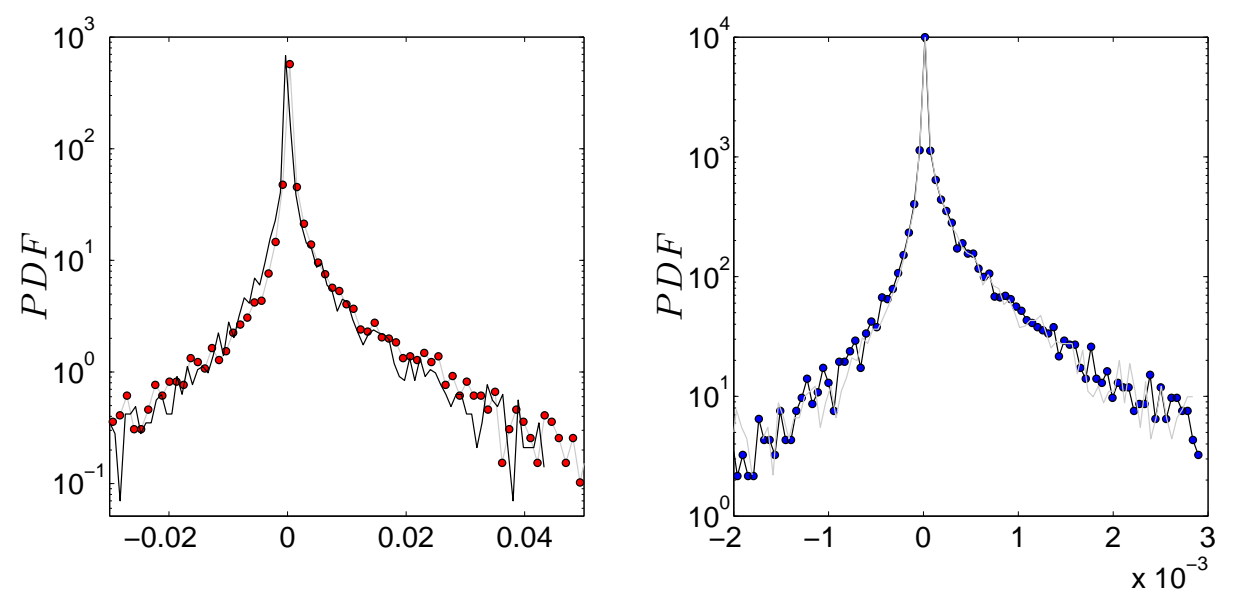

Figure 4: PDF of actual (solid line) and modeled (symbols) productions of RUM temperature variance (left) and RUM kinetic energy (right), at the periphery of the jet, at $t=6.2 T_{f} @$.

detailed in Ref. [25]. The evaluation of the accuracy of the models is performed at tensor/vector level by assessed each component of the RUM-ST and the RUM-HF against the actual quantity provided by the particle Eulerian database. Moreover, an assessment at scalar level is also given. Figure 1 shows the mean profiles of the RUM-ST components as predicted by 2QEASM compared with the actual stresses. Modeled, by $2 \Phi E A H F M$, and actual mean profiles of the RUM-HF components are depicted in Figure 2. For both the second-order moments, results at tensor/vector level are very satisfactory. At scalar level, the scalar quantities investigated are the productions of the RUM kinetic energy and the RUM temperature variance (first terms on the r.h.s. of Eqs. (8) and (9), respectively). Figure 3 shows the mean profiles of the two productions. In Figure 4, their PDFs evaluated at the periphery of the jet are depicted. They are computed by multiplying the local value of each modeled production by the actual-to-modeled magnitude ratio; in this way, only the shape of the PDFs is investigated [22]. Figure 3 shows that the two productions are successfully predicted, even if the RUM kinetic energy production is somewhat overestimated. Figure 4 shows that both the models are able to match the shape of the actual PDF and to reproduce reverse energy exchanges. Globally, results show that models compare very well with the Eulerian-Lagrangian DNS and properly reproduce all crucial trends of the computation. The proposed algebraic closures enable the ACBMM to correctly predict unsteady non-isothermal particle-laden turbulent flows, in dilute regime, for large Stokes number as well.

\section{Acknowledgements}

This work received funding from the European Community through the TIMECOP-AE project (Project \# AST5-CT-2006-030828). It reflects only the author's views and the Community is not liable for any use that may be made of the information contained therein. Numerical simulations were performed by the IBM Power6 machine; support from Institut du Développement et des Ressources en Informatique Scientifique (IDRIS) is gratefully acknowledged.

\section{References}

1. P. FÉvrier, O. Simonin And K. D. Squires. Partitioning of particle velocities in gas-solid turbulent flows into a continuous field and a spatially uncorrelated random distribution: theoretical formalism and numerical study. J. Fluid Mech. 533, 1-46, 2005. 
2. Q. WANG AND K. D. SQuires. Large eddy simulation of particle-laden turbulent channel flow. Phys. of Fluids 8(5), 1207-1223, 1996.

3. M. Boivin, O. Simonin And K. D. SQuires. On the prediction of gas - solid flows with two-way coupling using large eddy simulation. Phys. Fluids 12, 2080-2090, 2000.

4. V. Armenio, U. Piomelli, AND V. Fiorotto Effect of the subgrid scales on particle motion. Phys. of Fluids 11(10), 3030-3042, 1999.

5. P. FEDE AND O. Simonin. Numerical study of the subgrid fluid turbulence effects on the statistics of heavy colliding particles. Phys. Fluids 18, 045103, 2006.

6. S. E. Elghobashi And T. W. Abou-ARAB. A two-equation turbulence model for twophase flows. Phys. Fluids 26 (4), 931-938, 1983.

7. C. P. Chen AND P. E. Wood. A turbulence closure model for dilute gas-particle flows. The Canadian Journal of Chemical Engineering 63 (3), 349-360, 1985.

8. M. W. REEKS. On a kinetic equation for the transport of particles in turbulent flows. Phys. of Fluids 3(3), 446-456, 1991.

9. O. Simonin. Prediction of the dispersed phase turbulence in particle-laden jets., vol. 121, pp. 197-206. ASME FED, 1991.

10. L. I. ZAICHIK AND A. A. VinBerg. Modelling of particle dynamics and heat transfer in turbulent flows using equations for first and second moments of velocity and temperature fluctuations. In Proceedings of the Eighth Symposium on Turbulent Shear Flows (Munich, $F R G, 1991)$, pp. 1021-1026, 1991.

11. O. A. DRUZhinin AND S. Elghobashi. Direct numerical simulations bubble-laden turbulent flows using the two-fluid formulation. Phys. Fluids 10, 685-697, 1998.

12. J. FERRY AND S. BALACHANDAR. A fast Eulerian method for dispersed two-phase flow. Int. J. Multiphase Flow 27, 1199-1226, 2001.

13. S. L. RANI AND S. BALACHANDAR. Evaluation of the equilibrium Eulerian approach for the evolution of particle concentration in isotropic turbulence. Int. J. Multiphase Flow 29, 1793-1816, 2003.

14. J. FERRY AND S. BALACHANDAR. Equilibrium Eulerian approach for predicting the thermal field of a dispersion of small particles. Int. J. of Heat and Mass Transfer 48, 681689, 2005.

15. B. ShOtorban AND S. BALACHANDAR. A Eulerian model for large-eddy simulation of concentration of particles with small stokes numbers. Phys. Fluids 19, 118107, 2007.

16. A. Ferrante And S. Elghobashi. On the accuracy of the two-fluid formulation in direct numerical simulation of bubble-laden turbulent boundary layers. Phys. Fluids 19 (4), 045105, 2007.

17. M. Moreau, O. Simonin ANd B. BÉdAT. Development of gas-particle Euler-Euler LES approach: A priori analysis of particle sub-grid models in homogeneous isotropic turbulence. Flow, Turbulence and Combustion 84 (2), 295-324, 2010.

18. E. MASI, O. SimOnin AND B. BÉDAT. The mesoscopic Eulerian approach for evaporating droplets interacting with turbulent flows. Flow, Turbulence and Combustion 86, 563-583, 2011.

19. O. Simonin, P. FÉVRIER AND J. LAVIÉville. On the spatial distribution of heavy particle velocities in turbulent flow: From continuous field to particulate chaos. Journal of Turbulence 3 (N40), 2002.

20. L. SCHILler AND A. NAUmAn. A drag coefficient correlation. V.D.I. Zeitung 77, 318 320, 1935.

21. W.E. Ranz And W.R. Marshall. Evaporation from drops: II. Chem. Engng. Prog., 
48, 173180, 1952.

22. M. Moreau. Modélisation numérique directe et des grandes échelles des écoulements turbulents gaz-particules dans le formalisme eulérien mésoscopique. PhD thesis, Institut National Polytechnique de Toulouse, http://ethesis.inp-toulouse.fr/archive/00000434/, 2006.

23. E. MASI. Theoretical and numerical study of the modeling of unsteady non-isothermal particle-laden turbulent flows by an Eulerian-Eulerian approach. $\mathrm{PhD}$ thesis, Institut National Polytechnique de Toulouse, http://ethesis.inp-toulouse.fr/archive/00001256/, 2010.

24. J. Dombard, B. Leveugle, L. Selle, J. Reveillon, T. Poinsot and Y. D'ANGELO. Modeling heat transfer in dilute two-phase flows using the mesoscopic Eulerian formalism. International Journal of Heat and Mass Transfer 55 (5-6), 1486-1495, 2012.

25. A. Kaufmann, M. Moreau, O. Simonin and J. Helie. Comparison between Lagrangian and mesoscopic Eulerian modelling approaches for inertial particles suspended in decaying isotropic turbulence. J. Comput. Phys. 227 (13), 6448-6472, 2008.

26. R.M.C. So, L.H. Jin AND T.B. GATSKI. An explicit algebraic Reynolds stress and heat flux model for incompressible turbulence: Part II Buoyant flow. Theoret. Comput. Fluid Dynamics 17, 377406, 2004.

27. T. B. Gatski And C.G. Speziale. On explicit algebraic stress models for complex turbulent flows. J. Fluid Mech. 254, 59-78, 1993.

28. S. S. GiRimaji. Fully explicit and self-consistent algebraic Reynolds stress model. Theoretical and Computational Fluid Dynamics 8 (6), 387-402, 1996.

29. S. WAllin AND A.V. JohansSOn. An explicit algebraic Reynolds stress model for incompressible and compressible turbulent flows. J. Fluid Mech. 403, 89-132, 2000.

30. S. B. POPE A more general effective-viscosity hypothesis. J. Fluid Mech. 72 (02), 331340, 1975.

31. A. J. M. SPENCER. Theory of Invariants. In Continuum Physics. vol I, A.C. Eringen Editor, Academic Press, NewYork, 1971.

32. E. Masi, E. Riber, P. Sierra, O. Simonin And L. Gicquel. Modeling of the randomuncorrelated velocity stress tensor for unsteady particle Eulerian simulationin turbulent flows. Proceedings 7th International Conference on Multiphase Flow - ICMF 2010, http://ufdc.ufl.edu/UF00102023/00060, 2010.

33. P. SierRa SÁnCHEZ. Modeling the dispersion and evaporation of sprays in aeronautical combustion chambers. $\mathrm{PhD}$ thesis, Institut National Polytechnique de Toulouse, http://ethesis.inp-toulouse.fr/archive/00001839/, 2012.

34. P. M. Wikström, S. WALlin And A.V. JohansSOn. Derivation and investigation of a new explicit algebraic model for the passive scalar flux. Phys. of Fluids 12(3), 688-702, 2000.

35. S.B. Pope. Turbulent Flows. Cambridge University Press, 2000.

36. B. BÉDAT AND O. SimONin. Numerical study of the inter-phase heat transfers in a nonisothermal particle laden slab flow. Proceedings 5th International Conference on Multiphase Flow - ICMF 2004, No. 442, 2004.

37. O. Vermorel, B. Bédat, O. Simonin and T. Poinsot. Numerical study and modelling of turbulence modulation in a particle laden slab flow. Journal of Turbulence, 4 , N25, 2003.

38. E. Deutsch And O. Simonin. Large eddy simulation applied to the motion of particles in stationary homogeneous fluid turbulence. In Turbulence modification in Multiphase flow, ASME FED, vol. 110, pp. 35-42, 1991. 